\title{
Performance analysis of partial transmission cooperative strategy with unreliable relays
}

\author{
Yuanhao Liu* (D), Tao Shang*, Yan Gao and Zanyang Dong
}

\begin{abstract}
The cooperative communications show the great advantage on the relays that can share their resource to improve the decoding of destination. However, relays are considered not reliable due to a low signal strength or high interference, where the relays only transmit a fraction of the message. To solve this problem, in this work, the block processing are implemented in partial transmission (PT), which allows the relays to divide the source information into small blocks and calculate the reliability information of the message. In the destination, a correlated spatial reception scheme is designed to jointly decode several information copies. The motivation is that even if each relay may not decode the message completely correct, the relay can still improve the decoding of destination. This paper formulates the end-to-end outage probability of PT with unreliable relays. Simulation shows the proposed strategy can provide a significant improvement over the conventional strategies with unreliable relays.
\end{abstract}

Keywords: Cooperative communications, Outage probability, Unreliable relay

\section{Introduction}

Cooperative communications, as an emerging concept for wireless networks $[1,2]$, have received the significant attention recently. As a distributed transmission, the cooperative communications enable the neighboring nodes to share the resources to participate in the information transmission, through which the relays provide the spatial diversity as well as multiplexing gain for the destinations. Therefore, cooperative communications improve system performance and robustness of wireless networks, especially in some severe attenuation environments.

The idea of cooperative communications can be traced back to 1979 [1]. In recent years, various cooperative relaying protocols such as amplify-and-forward (AF) relaying strategy [2], decode-and-forward (DF) relaying strategy $[3,4]$, and their successors are proposed for wireless networks. Most of them adopt either maximal ratio combining or equal gain combining at the destination. Some work of them utilizes optimum power and time allocation [5], soft information [6], or global information [7] to get the performance improvements. The concept of relaying has been integrated into the fifth generation (5G) communication [8] and cellular structure [9], which has been

\footnotetext{
* Correspondence: 562675696@qq.com; shtsun_sjtu@hotmail.com School of Telecommunications Engineering, Xidian University, Taibai South Road, Xi'an 710071, China
}

written in the World interoperability for Microwave Access (WiMAX) standard [10] and Wireless World Initiative New Radio (WINNER) standard [11, 12]. The relay selection techniques have also been studied extensively, and several selection criteria that select the "reliable" relays have been proposed $[2,8,13,14]$. Moreover, cooperation techniques have also been considered in some latest communication standards such as IEEE 802.11s standard [6], IEEE 802.16j standard [10], and 3GPP's Long Term Evolution (LTE)-Advanced standard [15].

The relay's ability to reliably forward the message determines the performance of cooperative communications. In relay's AF strategy, the relays amplify the signal and then forward the message originally [16]. The message as well as the noise is sent together with the amplified signal strength; hence, the SNR at the destination is not improved. While in relay's conventional DF strategy, the relays need to decode the messages before they forward the messages, where the SNR at receiver can be improved [17]. Then, the selective decode-and-forward (SDF) strategy [2] is proposed. The SDF falls back to direct transmission if the relay cannot decode, where the energy can be saved compared to DF. Moreover, the concept of lossy forward (LF) is proposed, where the relays always forward the message with its soft information on each bit, even though it may contain errors 
$[6,18]$. With the LF scheme, the destination always receives the most useful information.

In this paper, we consider the situation where the relays have a high probability of failing in decoding the message due to the severe attenuation in channel or low SNR of the source. In AF, DF, and LF, the wrong message transmitted results in unwanted resource assumption. And in SDF, the solution to this problem is to let the relay make the decision whether its output is correct or not and discard it if it turns out to be erroneous. However, this may result in losing useful information that may help the destination's decoding, because the discarded information sequences have high probability to be decoded partly correct.

In this paper, we analyze the partial transmission (PT) cooperative strategy, which can overcome the shortcomings aforementioned. Compared to LF, PT allows relays to discard the bits with low soft information according to the channel state, which in fact allows the relays to make the decision for the destination. Moreover, the saved power helps to increase the transmit SNR for the useful information. Assuming that the power assumption to send every bit is identical, we implement the optimal block process, where the transmitted information from source is divided into a certain amount of information blocks in relay. The relays obtain the reliability information of the transmitted information bits by implementing the soft-input soft-output (SISO) decoders. With the channel state information (CSI) at receivers, the relays are able to calculate the optimal number of the correctly decoded information blocks. Consequently, the relays only forward the information blocks with low error probability. Correspondingly, the destination employs block combining to jointly decode the message. We derive the end-to-end outage probability of the proposed strategy as a function of the transmission rate and SNR with M-relaying. In addition, the numerical simulations are carried out in both one-relaying system and two-relaying system. Continuous changing channels show the advantage of the proposed strategy. The results show that the proposed strategy can get more diversity gain from the relay-to-destination channel and provide a significant improvement over the conventional strategies, particularly when the relay cannot reliably decode the message. We note that the more information transmission rate and channel gain from relay to destination are, the greater improvement PT can get.

The remainder of the paper is organized as follows. Section 2 describes the system model. Section 3 presents the outage probabilities of the proposed strategy. Section 4 gives numerical results and discussions. Section 5 concludes the paper.

\section{System model}

In this framework, the communication is considered to be cluster-crossed. Sources are either base station, head

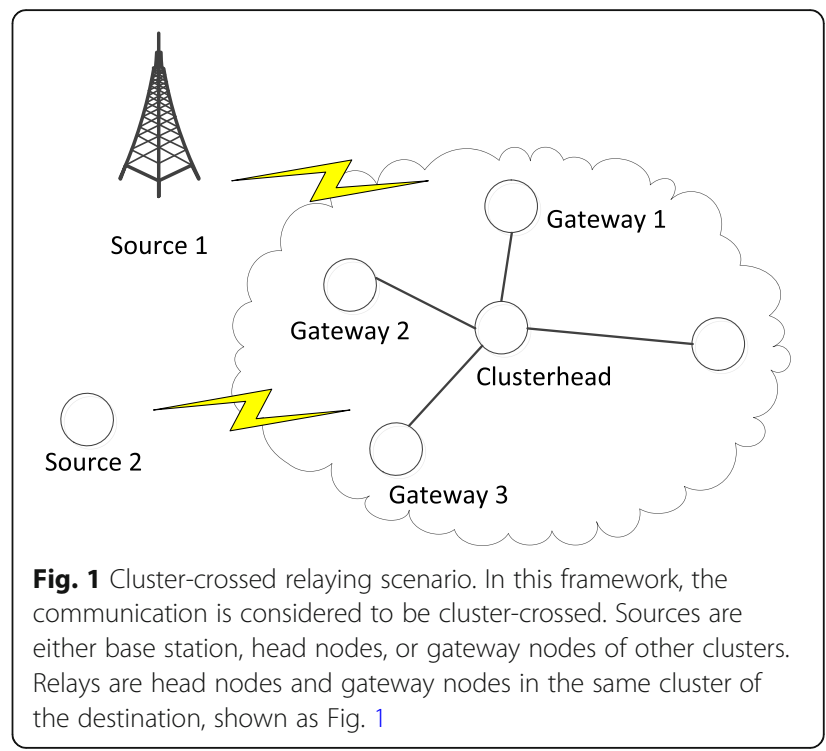

nodes, or gateway nodes of other clusters. Relays are head nodes and gateway nodes in the same cluster of the destination $[19,20]$, shown as Fig. 1.

In this paper, the strategies are analyzed in the system with the identical power consumption for fairness. We consider the narrowband transmissions in the wireless network in which the channels between any two nodes are subject to the effects of slow-flat Nakagami-m fading with Nakagami factor $m_{\mathrm{i}}$ and additive white Gaussian noise (AWGN). Nodes in the network work in a half-duplex mode where they cannot transmit and receive simultaneously in the same

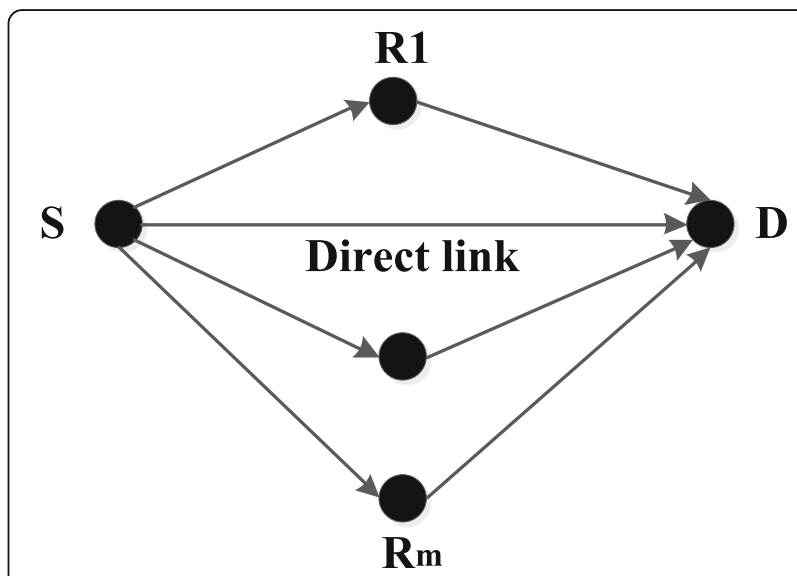

Fig. 2 Simplified system model considering one source, $m$ relays, and one destination. The figure shows the system model. In this work, the transmission process is divided into two phases, phase I and phase II. In phase I, an information source broadcasts the message and multiple relays and the destination receive the message. In phase II, according to the proposed relaying strategy, the multiple relays transmit the received message to the destination. After receiving several copies of the information sequences, the destination jointly decode the messages 
time slot. We assume that CSI is only known at the receivers and all the transmitters are assigned with an averaging time slot. After the destination receives several copies of the information sequences, it implements a novel spatial reception scheme, block combining, to jointly decode the message.

Similar to $[5-8,16]$, we consider a cooperative wireless network that consists of one source node $S$, one destination node $\mathrm{D}$, and $\mathrm{M}$ relay nodes. The simplified system model is shown as Fig. 2. The squared envelope of the channel gains associated with source-destination, source-relay, and relay-destination links are denoted by $\gamma_{\mathrm{sd}}, \gamma_{\mathrm{sr}_{\mathrm{i}}}$, and $\gamma_{\mathrm{r}_{\mathrm{i}}}$, respectively. These squared envelope of the channel gains are independent exponentially distributed random variables (RVs) with mean values $\bar{\gamma}_{\mathrm{sd}}$, $\bar{\gamma}_{\mathrm{sr}_{\mathrm{i}}}$, and $\bar{\gamma}_{\mathrm{r}_{\mathrm{i}} \mathrm{d}}$. The amplitudes of the channel gains of source-destination links, source-relay links, and relay-destination links follow independent Nakagami-m distribution. The cooperative strategy is described as two phases. In phase I, the source transmits information signal to the destination in the first time slot, and the signal is also received by the relays as well. In phase II, the relays help transmitting the received message in $M$ equal time slots respectively.

More specifically, we assume $b$ bit information every time transmitted from the source are divided into $N$ information blocks, and each block contains $L$ bit information. The information is modulated by BPSK.

In phase I, the source broadcasts its information signal $x$ to the destination and all the relays. The received signals $y_{\text {sd }}$ and $y_{\mathrm{Sr}_{\mathrm{i}}}$ at the destination and the relays can be written as:

$$
\begin{aligned}
& y_{\mathrm{sd}}=\sqrt{P_{\mathrm{sd}}} h_{\mathrm{sd}_{\mathrm{d}}} x+n_{\mathrm{sd}} \\
& y_{\mathrm{sr}_{\mathrm{i}}}=\sqrt{P_{\mathrm{sr}_{\mathrm{i}}}} h_{\mathrm{sr}_{\mathrm{i}}} x+n_{\mathrm{sr}_{\mathrm{i}}}
\end{aligned}
$$

where $P_{\mathrm{sd}}=P_{\mathrm{sr}_{\mathrm{i}}}=\frac{P}{M+1}$, which is the transmission power in phase I. In (1) and (2), $n_{\mathrm{sd}}$ and $n_{\mathrm{sr}_{\mathrm{i}}}$ are the corresponding received AWGN at the destination and the relays with mean zero and variance $N_{0} \cdot h_{\mathrm{sd}}$ and $h_{\mathrm{sr}_{\mathrm{i}}}$ are the channel coefficients from the source to the destination and the $i$-th relay, respectively. The fraction $\frac{1}{M+1}$ is the fact that the power $P$ of each transmission is averaged by one source and $M$ relays. The bandwidth of the whole cooperation system is normalized. In the whole process, each node needs some certain amount of time slots, which is $\frac{1}{M+1}$. The received SNR, $\mathrm{SNR}_{\mathrm{sr}_{\mathrm{i}}}$ and $\mathrm{SNR}_{\mathrm{sd}}$ at the relay and the destination, can be expressed as:

$$
\begin{aligned}
\mathrm{SNR}_{\mathrm{sr}_{\mathrm{i}}} & =\frac{\frac{P}{M+1}}{N_{0}}\left|h_{\mathrm{sr}_{\mathrm{i}}}\right|^{2}=\rho \gamma_{\mathrm{sr}_{\mathrm{i}}} \\
\mathrm{SNR}_{\mathrm{sd}} & =\frac{\frac{P}{M+1}}{N_{0}}\left|h_{\mathrm{sd}}\right|^{2}=\rho \gamma_{\mathrm{sd}}
\end{aligned}
$$

and $\rho=\frac{P}{M+1} / N_{0}$, which is the transmission SNR of the source node.

In phase II, the relays transmit the message to the destination with the signal $\hat{\mathrm{x}}_{\mathrm{i}}$, and the transmission power of the relay is $P_{\mathrm{r}_{\mathrm{i}}}$. The received signal $y_{\mathrm{r}_{\mathrm{i}} \mathrm{d}}$ at the destination can be written as

$$
y_{\mathrm{r}_{\mathrm{i}} \mathrm{d}}=\sqrt{P_{\mathrm{r}_{\mathrm{i}}}} h_{\mathrm{r}_{\mathrm{i}} \mathrm{d}} \hat{\mathrm{x}}_{\mathrm{i}}+n_{\mathrm{r}_{\mathrm{i}} \mathrm{d}}
$$

where $n_{\mathrm{r}_{\mathrm{i}} \mathrm{d}}$ is the corresponding received noise at destination, and $h_{\mathrm{r}_{\mathrm{i}} \mathrm{d}}$ is the channel coefficients from the relays to the destination.

\section{Outage probability}

In this section, we evaluate the end-to-end outage probabilities of the proposed strategy based on multiple relays.

\subsection{Proposed strategy}

In the proposed partial transmission strategy cooperative, the relays are capable of getting the soft information of each bit with SISO decoder. After block process and some calculation, the relays transmit the selected information blocks which have lower error probability to the destination. The relaying process is shown in Fig. 3.

In phase I, $\mathrm{SNR}_{\mathrm{sr}_{\mathrm{i}}}$ is the Nakagami- $m$ distributed RVs. The PDF of $\mathrm{SNR}_{\mathrm{Sr}_{\mathrm{i}}}$ can be written as:

$$
f_{\mathrm{SNR}_{\mathrm{s}_{\mathrm{i}}}}(x)=\frac{2 m_{i} \cdot\left(\lambda_{x}\right)^{m_{\mathrm{i}}}}{\Gamma\left(m_{i}\right)} \cdot x^{2 m_{i}-1} \cdot \exp \left(-\lambda_{x} \cdot m_{i} x^{2}\right)
$$

where $\Gamma(\cdot)$ is gamma function and $m_{i}$ is the Nakagami parameter.

$$
\lambda_{x}=\frac{1}{\rho \bar{\gamma}_{\mathrm{sr}_{\mathrm{i}}}}
$$

The information is modulated by BPSK, and we assume that symbols 0 and 1 are modulated into 1 and -1 , respectively. The conditional BER of BPSK is given by:

$$
\mathrm{BER}_{\mathrm{sr}_{\mathrm{i}}}=\frac{1}{2} \operatorname{erfc}\left(\sqrt{\mathrm{SNR}_{\mathrm{sr}_{\mathrm{i}}}}\right)
$$

\subsubsection{Block error probability}

We implement the SISO decoder and the average bit error probability scheme to get the reliability information. For a $L$ bit information sequence, the SISO decoder outputs the reliability information of the inputs, usually in the form of the log-likelihood ratio (LLR) [21].

$$
L\left(u_{i}\right)=\ln \frac{P\left(u_{i}=+1 \mid x\right)}{P\left(u_{i}=-1 \mid x\right)}
$$

where $u_{i}$ is hard-output of the $i$-th bit. The relay can get the bit error probability as: 


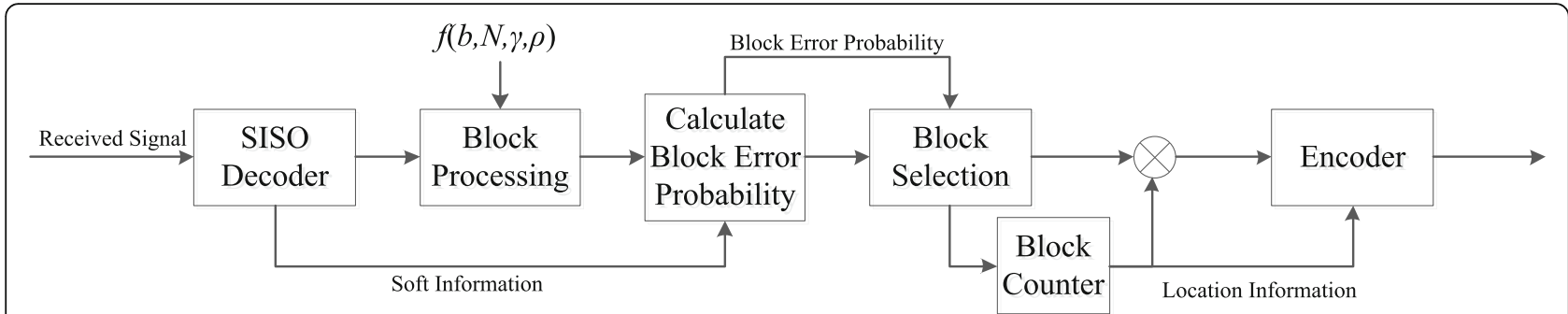

Fig. 3 Partial transmission at relay. The figure shows the detailed partial transmission at relay. After the relay received signal, it uses the SISO decoder to calculate the soft information of each bit, then the block error probability in the third step. The relay can also get the optimal block size with the information bits, the received SNR, and the average channel gain in the second step. Using the calculation results, several blocks with lower error probability are selected, then encoded with their location information, where the location information is encoded in the imaginary part of the signal

$$
P_{\mathrm{bit}}=\frac{1}{1+e^{\left|L\left(u_{i}\right)\right|}}
$$

For simplicity, the information sequence is divided into $N$ blocks, and the length of each block is $L$ bits.

There are two different schemes to calculate the error probability of block. One is that the block is considered erroneous as long as 1 bit information is wrong. We have the error probability of block as $\left[1-\prod_{i=1}^{L}\left(1-P_{\mathrm{bit}}\right)\right]$, where the major drawback is that the block is discarded even the decoding is correct with 1 bit error probability being too high. The other one is that the error probability of block is calculated as average bit error probability, in which $P_{\mathrm{b}}$ can be expressed as [22].

$$
P_{\mathrm{b}}=\frac{1}{L} \sum_{i=1}^{L} \frac{1}{1+e^{\left|L\left(u_{i}\right)\right|}}
$$

In this paper, we adopt the second scheme to calculate the error probability of block.

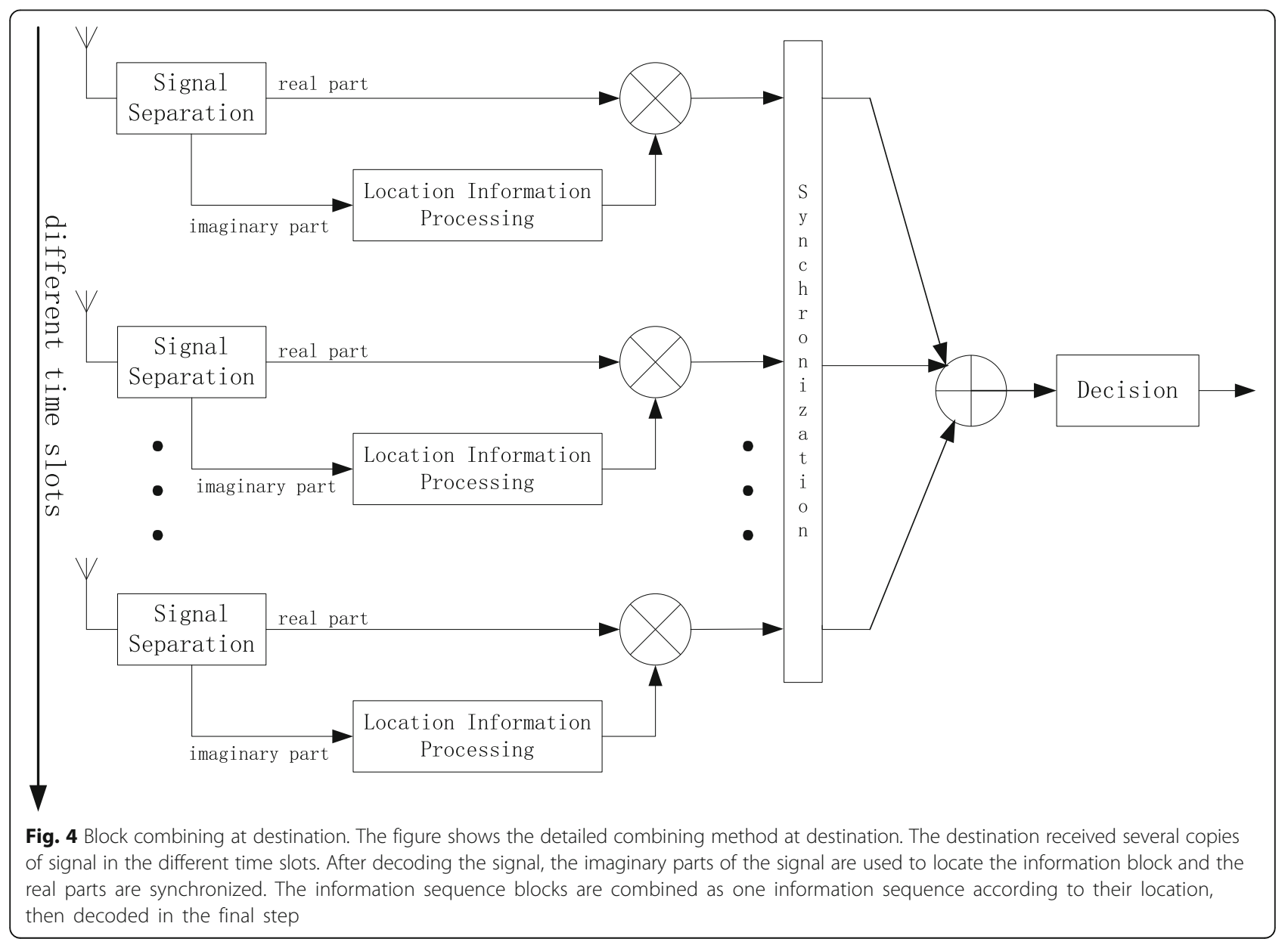




\subsubsection{Block processing}

A sequence with $b$ bit information is divided into $N$ equal blocks. After the relay gets the error probability of block, the relay regards several blocks who have the lowest error probability as the correct blocks [18], and the number of those blocks is $N_{\mathrm{r}_{\mathrm{i}}}$, which is the optimal transmitted block number of the $i$-th relay. $N_{\mathrm{r}_{i}}$ can be approximated by the $\mathrm{BER}$ at the relay, which can be formulated as:

$$
N_{\mathrm{r}_{i}}=\left\lfloor\frac{b \cdot\left(1-\mathrm{BER}_{\mathrm{sr}_{i}}\right)}{L}\right\rfloor=\left\lfloor N \cdot\left(1-\mathrm{BER}_{\mathrm{sr}_{i}}\right)\right\rfloor
$$

where the function $\lfloor\phi\rfloor$ returns the largest integer no greater than $\phi, \mathrm{BER}_{\mathrm{sr}_{i}}$ is the received BER at the $i$-th relay.

In order to maintain the equal length of the $(M+1)$ copies, the incorrect blocks at each relay are replaced by equilong blanks.

If relays discard any useful information, they have to tell the destination which bits are discarded. Thus, the relays must add extra location information. The additional information is done in the complex field [6].The number of additional location information for each information block is $\left\lceil\log _{2}(\mathrm{~N})\right\rceil$ bits; thus, the number of location information bits, which is required by the relay to send $N_{\mathrm{r}_{i}}$ information blocks, is $\left\lceil\log _{2}(N)\right\rceil \cdot\left\lfloor\mathrm{N} \cdot\left(1-\mathrm{BER}_{\mathrm{sr}_{i}}\right)\right\rfloor$.

The size of each block should be as small as possible; thus, the relay is able to reserve more useful bits. The smallest block scheme among them is 1 bit per block. If the relay discards any bit, it has to tell the destination the location information, which consists $\left\lceil\log _{2}(\mathrm{~N})\right\rceil$ bits for a $N$ block information sequence. However, in order to reduce the additional information, we may need the bigger blocks.

Therefore, there is a tradeoff between the wrong bits accuracy and the additional information, where the former one requires smaller block size while the latter one needs bigger block size. In this paper, we intend to achieve the lowest end-to-end outage probability, so the block size is set as small as possible with the constraint of the finite power consumption.

In this work, it is assumed that the power assumption to send every bit is identical, and the above problem can be described as follows. The allotted power of the $i$-th relay is $P_{\mathrm{r}_{i}}$, which can be divided into two parts in PT, $P_{\mathrm{rx}_{i}}$ and $P_{\mathrm{dw}_{i}} . P_{\mathrm{rx}_{i}}$ is the power required to send the information blocks, and it is allotted in direct proportion to $N_{\mathrm{r}_{i}} . P_{\mathrm{dw}_{i}}$ is the power required to send the location information bits. The $P_{\mathrm{r} x_{i}}$ and $P_{\mathrm{dw}_{i}}$ can be derived as:

$$
P_{\mathrm{r} x_{i}}=\frac{P}{1+M} \cdot \frac{1}{N} \cdot\left\lfloor N \cdot\left(1-\mathrm{BER}_{\mathrm{sr}_{i}}\right)\right\rfloor
$$

$$
P_{\mathrm{dw}_{i}}=\frac{P}{1+M} \cdot \frac{1}{b} \cdot\left\lceil\log _{2}(N)\right\rceil \cdot\left\lfloor\mathrm{N} \cdot\left(1-\mathrm{BER}_{\mathrm{sr}_{i}}\right)\right\rfloor
$$

Obviously, we get the constraint $P_{\mathrm{r}_{i}}+P_{\mathrm{dw}_{i}}<\frac{P}{1+M}$, and after transformation, it can be simplified as $\frac{\left\lfloor N \cdot\left(1-\mathrm{BER}_{\mathrm{sr}_{i}}\right)\right\rfloor}{\mathrm{N}}$ $+\frac{\left\lceil\log _{2}(N)\right\rceil \cdot\left\lfloor N \cdot\left(1-\mathrm{BER}_{\mathrm{sr}_{i}}\right)\right\rfloor}{\mathrm{b}}<1$. Hence, for the $i$-th relay, the optimal block size can be calculated as:

$$
\begin{aligned}
& \underset{N}{\arg \max } f(b, N, \gamma, \rho) \\
& s \cdot t \cdot f(b, N, \gamma, \rho) \leq 1
\end{aligned}
$$

where $f(b, N, \gamma, \rho)=\frac{\left\lfloor N \cdot\left(1-\mathrm{BER}_{\mathrm{sr}_{i}}\right)\right\rfloor}{N}+\frac{\left\lceil\log _{2}(N)\right\rceil \cdot\left\lfloor N \cdot\left(1-\mathrm{BER}_{\mathrm{sr}_{i}}\right)\right\rfloor}{b}$.

The optimization is to find the maximal number of the blocks, or the minimal block size, to meet the constraint of the transmission power at the relay. The constraint is in practice that each relay discards some of the received bit sequence, and the location information should be set as no bigger than that, because the power consumption of each relay is pre-allocated.

However, $f(b, N, \gamma, \rho)$ is a non-convex function, which is hard to obtain the derived function. Therefore, we optimize the number of blocks of each relay by one dimensional iteration method in Algorithm 1.

The proposed algorithm divides the optimization into two local problems. Therefore, there exist two loops to optimize the function in Algorithm 1. The first loop employs the doubling step length search algorithm to find the upper boundary. The set of max iterations of seeking boundary avoids the situation where the function $f(b$, $N, \gamma, \rho)$ never meets the restricted condition. The doubling step length search algorithm is easily achieved, the time complexity of which is $\mathrm{O}(\log \mathrm{N})$. The second loop exploits bisearch algorithm to optimize the number of blocks, and the time complexity of bisearch algorithm is $\mathrm{O}(\log \mathrm{N})$. Then, the time complexity of entire optimization is also $\mathrm{O}(\log N)$. During the whole optimization, the relays have to storage information bits, average $S-R$ channel gain, transmit power of source node, cutoff accuracy, max iterations of seeking boundary, blocks number and function output. They are iterated as Algorithm 1 runs. Therefore, the space complexity is constant, i.e., $\mathrm{O}(1)$.

In phase II, the relays forward the message to the destination, and the received signal $y_{\mathrm{r}_{\mathrm{d}} \mathrm{d}}$ at the destination is given by (5). The destination node decodes the received several copies with the block combining, as shown in Fig. 4, where the destination combines the copies block by block by exploiting the location information. The location information processing outputs 1 for useful blocks and 0 for blanks, which intends to get rid of unwanted noise and helps the synchronization. Consequently, the erroneous blocks at relay have no contribution to the final decision. 


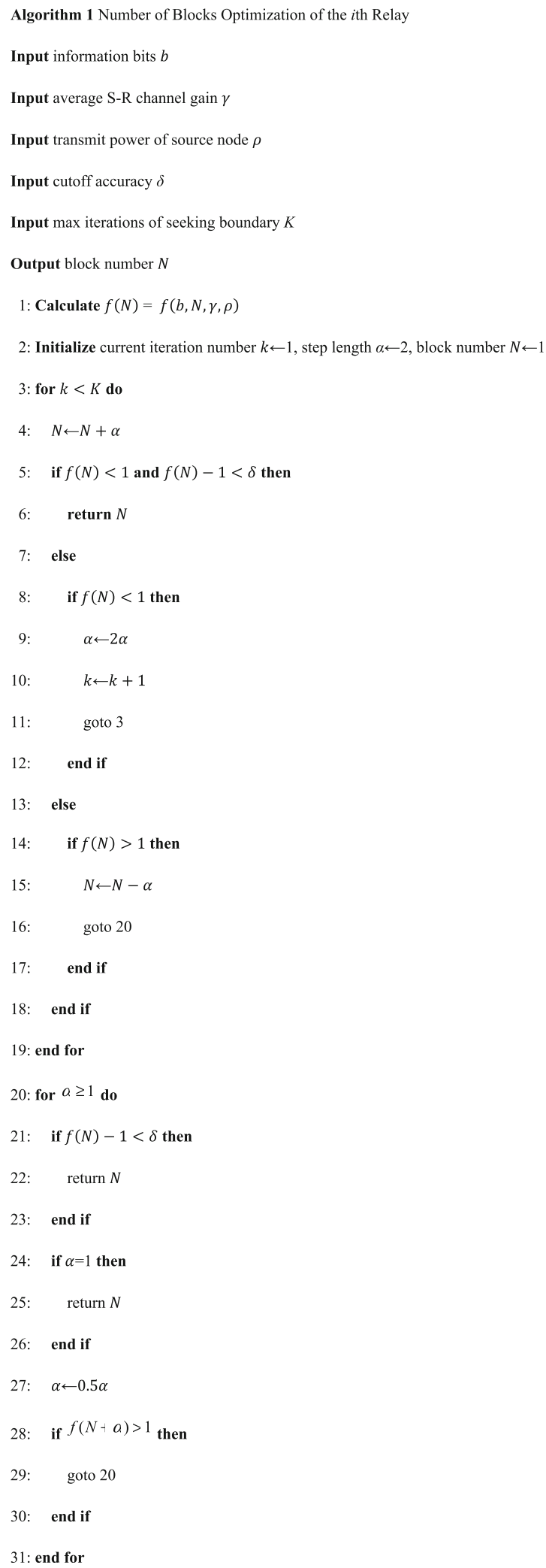

\subsubsection{End-to-end outage probability}

In phase II, after block combining, the received SNR at the destination from the $i$-th relay, $\mathrm{SNR}_{\mathrm{r}_{i} \mathrm{~d}}$, can be expressed as:

$$
\mathrm{SNR}_{\mathrm{r}_{i} \mathrm{~d}}=\frac{P_{\mathrm{rx}_{i}}}{N_{0}} \gamma_{\mathrm{r}_{i} \mathrm{~d}}=\frac{\rho}{N} \cdot\left\lfloor N \cdot\left(1-\mathrm{BER}_{i}\right)\right\rfloor \gamma_{\mathrm{r}_{i} \mathrm{~d}}
$$

The received signal at the destination $y_{\mathrm{r}_{i} \mathrm{~d}}$ in (5) can be transformed as:

$$
y_{\mathrm{r}_{i} \mathrm{~d}}=\sqrt{P_{\mathrm{r}_{i}}} h_{\mathrm{r}_{i} \mathrm{~d}} \hat{x}+n_{\mathrm{r}_{i} \mathrm{~d}}
$$

During the whole cooperative process, the destination has received $(M+1)$ copies of the message, so the accumulated mutual information at destination by block combining can be expressed as:

$$
I_{\mathrm{PT}}=\frac{1}{1+m} \log _{2}\left(1+\mathrm{SNR}_{\mathrm{sd}}+\sum_{i=1}^{M} \mathrm{SNR}_{\mathrm{r}_{i} \mathrm{~d}}\right)
$$

The fraction $\frac{1}{M+1}$ is the fact that each transmitter only use $\frac{1}{M+1}$ of the total time slots.

It is assumed that the transmission rate of the information is $R_{\mathrm{t}}$, and according to the information theory, the end-to-end outage probability $P_{\text {outPT }}$ can be expressed as:

$$
\begin{aligned}
& P_{\text {outPT }}=P\left(I_{\mathrm{PT}}<R_{\mathrm{t}}\right) \\
& =\operatorname{Prob}\left(\frac{1}{1+M} \log _{2}\left(1+\mathrm{SNR}_{\mathrm{sd}}+\sum_{i=1}^{M} \mathrm{SNR}_{\mathrm{r}_{\mathrm{i}} \mathrm{d}}\right)<R_{\mathrm{t}}\right) \\
& =\operatorname{Prob}\left(\mathrm{SNR}_{\mathrm{sd}}+\sum_{i=1}^{M} \mathrm{SNR}_{\mathrm{r}_{\mathrm{i}} \mathrm{d}}<2^{(1+M) R_{\mathrm{t}}}-1\right)
\end{aligned}
$$

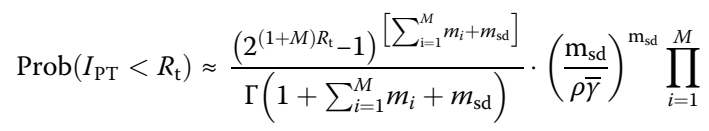

$$
\begin{aligned}
& \times\left(\frac{m_{i} N}{\rho\left\lfloor N \cdot\left(1-\mathrm{BER}_{\mathrm{sr}_{i}}\right)\right\rfloor \bar{\gamma}_{\mathrm{r}_{i} \mathrm{~d}}}\right)^{m_{i}}
\end{aligned}
$$

where $\mathrm{m}_{\mathrm{sd}}$ is the Nakagami parameter of the direct link. If without the direct link, (19b) turns into

$$
\operatorname{Prob}\left(I_{\mathrm{PT}}<R_{\mathrm{t}}\right) \approx \frac{\left(2^{M \cdot R_{\mathrm{t}}}-1\right)^{\sum_{i=1}^{M} m_{i}}}{\Gamma\left(1+\sum_{i=1}^{M} m_{i}\right)} \prod_{i=1}^{M}\left(\frac{m_{i} N}{\rho\left\lfloor N \cdot\left(1-\mathrm{BER}_{\mathrm{st}_{i}}\right)\right\rfloor \bar{\gamma}_{\mathrm{r}_{i} \mathrm{~d}}}\right)^{\mathrm{m}_{\mathrm{i}}}
$$

Proofs of (19b) and (20) are provided in Appendix.

\section{Outage probability analyses and discussions}

In this section, we analyze and compare four strategies, PT, SDF, AF, and direct transmission (DT), on the situation with one-relaying system and two-relaying system, i.e., $M=1$ and $M=2$ in the above analyses. For these 


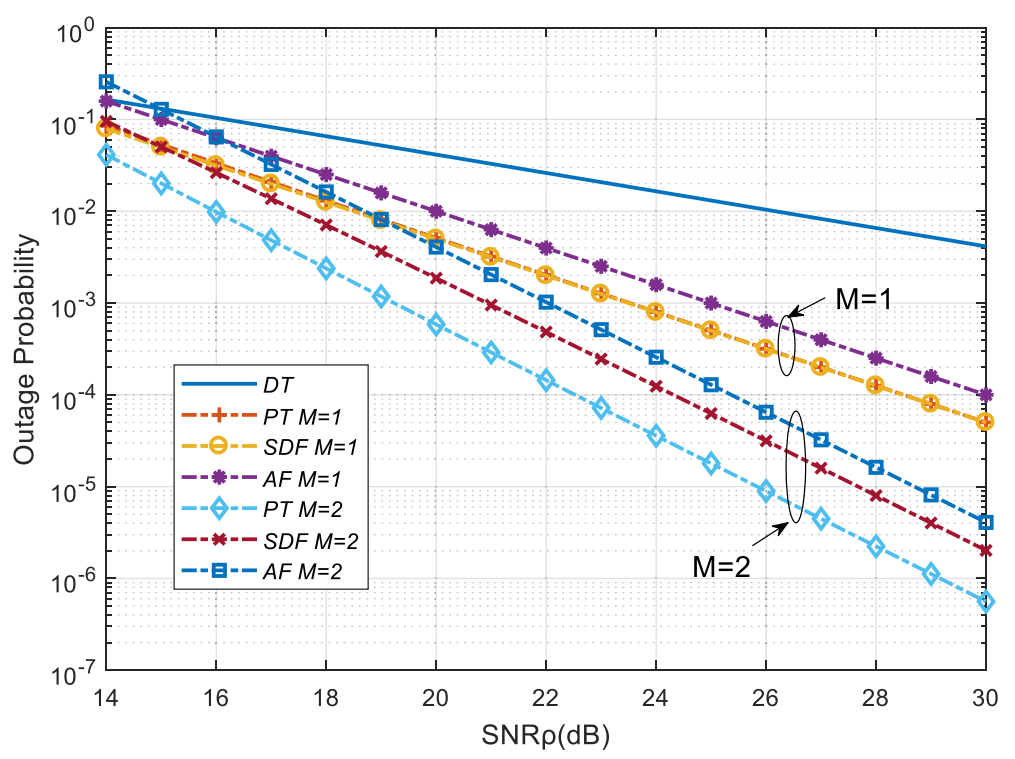

Fig. 5 Outage probability versus signal-to-noise ratio. The figure shows that the simulations on outage probability versus SNR of partial transmission (PT) and three conventional cooperative strategies, direction transmission (DT), selective decode-and-forward (SDF), and amplify-anddecode (AF) with the existence of direct link, on the situations with one-relaying system and two-relaying system. To simulate the unreliable relays, the simulation parameters are set as: the number of information bits is 100,000; the transmission rate of information is $0.5 \mathrm{~b} / \mathrm{s} / \mathrm{Hz}$; all the channel states from source to relays, relays to destination and source to destination are set the same, as 0.1. It can be seen in Fig. 5 that PT performs slightly worse than that of SDF before $\rho=24 \mathrm{~dB}$ when $M=1$, because in the poor channels, the relay can hardly help the decoding at destination. After, PT gets the lower outage probability compared with SDF. Because as SNR increases, the relay can transmit more information block in PT, while SDF still has high probability failing to decode at the relay. When $M=2$, PT gets better performance over the conventional strategies, because two relays can get double spatial diversity than that of the situation with $M=1$. As SNR increases, the improvement of PT gets bigger. It is also noted that at low SNR, more relays cost more power and bandwidth, but they are unable to help the destination in such a low channel gain of S-R links

four strategies, we do simulation works of the information outage probability with and without direct link respectively. We assume the number of information bits $b=100,000$ and the transmission rate of information $R_{\mathrm{t}}=0.5(\mathrm{~b} / \mathrm{s} / \mathrm{Hz})$.

Figures 5 and 6 show the end-to-end outage probability versus the transmission SNR $\rho$ at the source and information transmission rate $R_{\mathrm{t}}$ with direct link, respectively. For simplicity, the channel states of two relays are set as the same, which are expressed by channel gain variance as follows: $\bar{\gamma}_{\mathrm{sr} 1}=\bar{\gamma}_{\mathrm{sr} 2}=0.1, \bar{\gamma}_{\mathrm{sd}}$ $=0.1$, and $\bar{\gamma}_{\mathrm{rd} 1}=\bar{\gamma}_{\mathrm{rd} 2}=0.1$. The Nakagami parameters are set as: $m_{\mathrm{sd}}=m_{i}=1$. The above condition responds to the scenarios in all poor channels, where the relays are considered to be unreliable. We analyze the relationship between the outage probability and channel state of relay-to-destination links in Figs. 7 and 8, in the situation with and without direct link respectively. We then discuss the relaying performances without direct link in Fig. 9, to compare them comprehensively.

In Figs. 5 and 6, the channel states from $S$ to $R, R$ to $\mathrm{D}$, and $\mathrm{S}$ to $\mathrm{D}$ are the same. Figure 5 shows that the outage probability of PT performs almost the same to that of SDF when $M=1$. However, with the R-D channel gets better, PT gets better than SDF, which can be seen in Fig. 7. In the poor channels, the relay can hardly help the decoding at destination. After $\rho=24 \mathrm{~dB}$, PT gets the lower outage probability compared with SDF. Because as SNR increases, the relay can transmit more information block in PT, while SDF still has high probability failing to decode at the relay. When $\mathrm{M}=2$, PT gets better performance over the conventional strategies, two relays can get double spatial diversity than that of the situation with $M=1$. As SNR increases, the improvement of PT gets bigger. It is also noted that at low SNR, more relays cost more power and bandwidth, but they are unable to help the destination in such a low channel gain of $\mathrm{S}-\mathrm{R}$ links.

In Fig. 6, the transmission SNR $\rho=20 \mathrm{~dB}$. It can be seen that the outage probability gets higher as the information transmission rate increases. For the case of $\mathrm{M}=$ 2 at low $R_{\mathrm{t}}$, the PT performs worse than SDF and AF. Because the relays at low rate have high probability to decode the message or help the decoding at destination, so they are reliable relays, while the relays in PT 


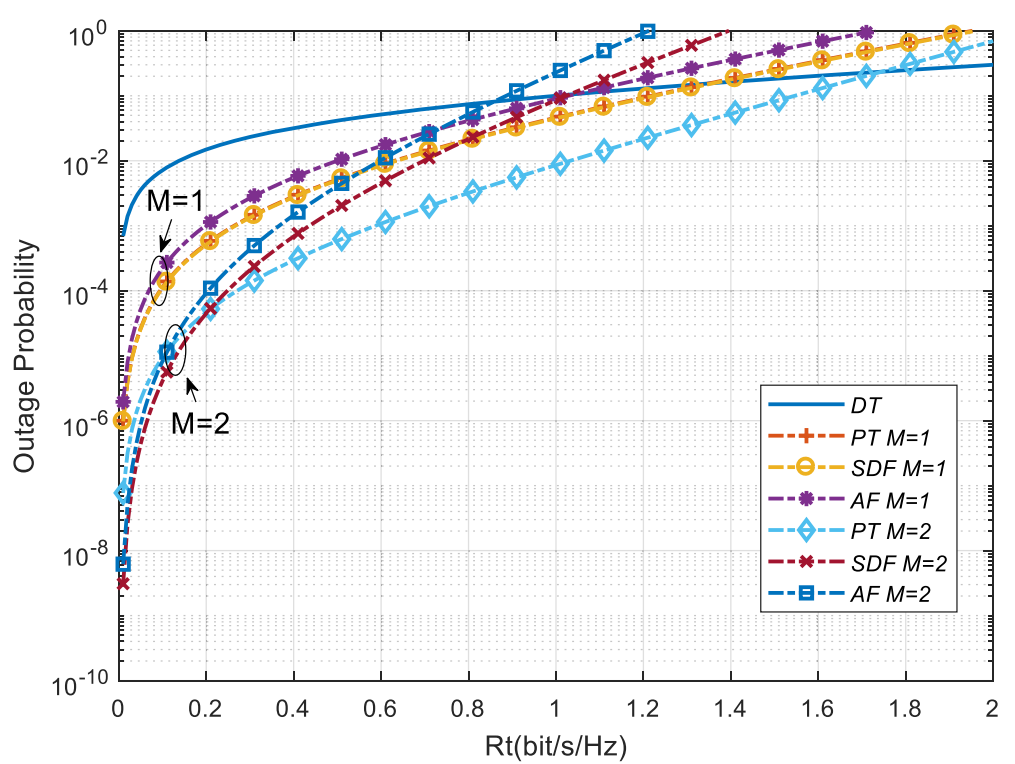

Fig. 6 Outage probability versus the information transmission rate. The figure shows that the simulations on outage probability versus transmission rate of partial transmission (PT) and three conventional cooperative strategies, direction transmission (DT), selective decodeand-forward (SDF), and amplify-and-decode (AF), on the situations with one-relaying system and two-relaying system. To simulate the unreliable relays, the simulation parameters are set as follows: the number of information bits is 100,000; the transmit SNR of source is $20 \mathrm{~dB}$; and all the channel states from source to relays, relays to destination, and source to destination are set the same, as 0.1 . It can be seen in Fig. 6 that the outage probability gets higher as the information transmission rate increases. For the case of two-relaying system at low transmission rate, the PT performs worse than SDF and AF. Because the relays at low rate have high probability to decode the message or help the decoding at destination, they are reliable relays, while the relays in PT cannot transmit all the information sequence. As transmission rate gets higher, the relays become unreliable, where the partial transmission shows the great advantage. However, if transmission rate rises to some certain threshold, all the relays are useless, so that the two-relaying system performs worse than the onerelaying system and even worse than the direct transmission. However, PT can postpone this threshold

cannot transmit all the information sequence. As $R_{\mathrm{t}}$ gets higher, the relays become unreliable, where the partial transmission shows the great advantage. However, if $R_{\mathrm{t}}$ rises to some certain threshold, all the relays are useless, so that the two-relaying system performs worse than the one-relaying system and even worse than the direct transmission. However, PT can postpone this threshold.

Figures 7 and 8 show the outage probability versus the channel gain between the relay and the destination in one-relaying system and two-relaying system, respectively.

Figure 7 shows that PT gets the better performance than the conventional strategies, as the R-D channel gain gets better than 0.1 with direct link and 0.128 without direct link. It is also shown that SDF and AF are not able to obtain the spatial diversity gain from the R-D channel, because they are limited by the poor channel ahead of the relay. For SDF, the state of the S-D channel determines the decoding ability of the relay. For PT, although $\mathrm{R}$ node fails to decode all the information correctly, the benefit of R-D channel still obtains diversity gain for the destination.

In Fig. 8, in order to facilitate the analysis, we assume that the two R-D channels follow the same distribution. PT gets better than SDF after about $\bar{\gamma}_{\text {rd }}=0.05$ and $\bar{\gamma}_{\text {rd }}=0.35$, in the situations with and without direct link respectively. As the channel gain between relays and destination increases, the gain value that PT gets better than SDF is nearly half of it in one-relaying system, where the certain amount of location information of PT degrades some performance with and without direct link. Generally speaking, when the R-D channel is better than the severely attenuated S-D channel, i.e., the relays are considered to be unreliable, the performance of PT is better than that of the conventional strategies.

Figure 9 shows the outage probability performances in the situation without direct link. Based on the discussion above, we set $\bar{\gamma}_{\text {rd }}=0.2$ in Fig. 9, and other parameters remain the same. Certainly, the existence of the direct link helps to gain more spatial diversity gain, which can be seen when compared to Fig. 5. At low SNR, Fig. 9 shows none of the mentioned strategies is reliable. As SNR increases, the improvements of PT are more obvious without direct link.

\section{Methods}

\subsection{Research object}

This work aims to solve the problem of unreliable relays. We designed the block processing at relaying 


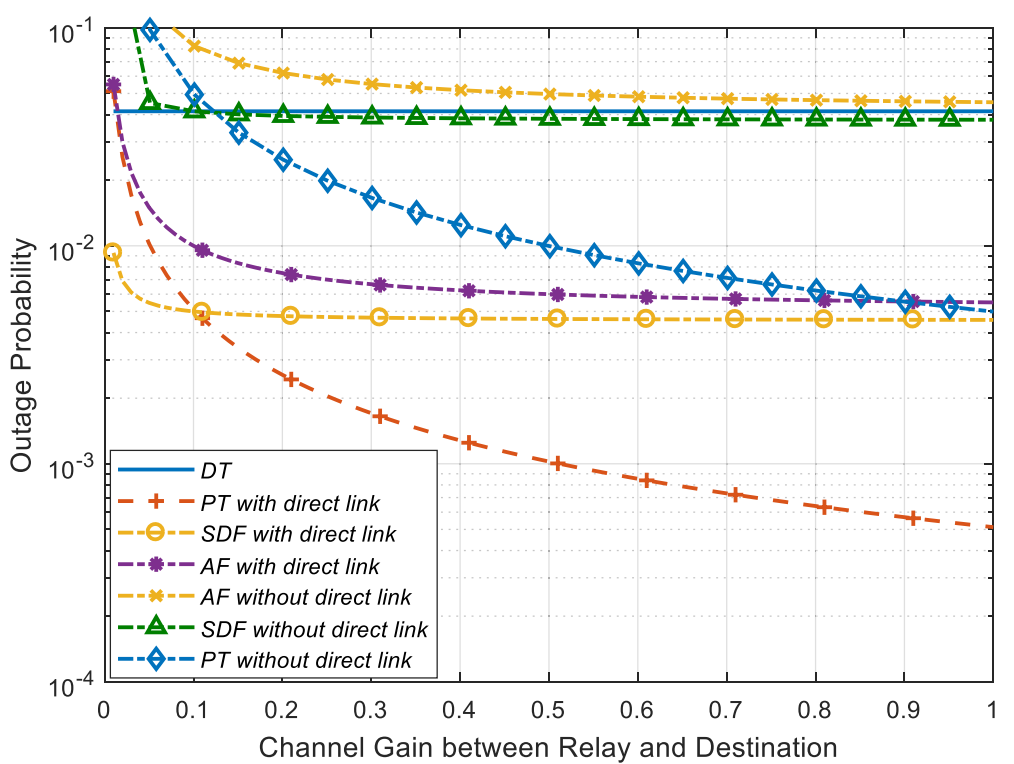

Fig. 7 Outage probability versus channel gain between relay and destination with one relay. Shows that the simulations on outage probability versus channel gain between relay and destination (R-D) of partial transmission (PT) and three conventional cooperative strategies, direction transmission (DT), selective decode-and-forward (SDF), and amplify-and-decode (AF), on the situations with one-relaying system. To simulate the unreliable relays, the simulation parameters are set as: the number of information bits is 100,000; the transmit SNR of source is $20 \mathrm{~dB}$; other channel states from relays to destination (R-D) and source to destination (S-D) are set the same, as 0.1 . The figure shows that PT gets the better performance than the conventional strategies as the R-D channel gain gets better than 0.1. It is also shown that SDF and AF are not able to obtain the spatial diversity gain from the R-D channel, because they are limited by the poor channel ahead of the relay. For SDF, the state of the S-D channel determines the decoding ability of the relay. For PT, although R node fails to decode all the information correctly, the benefit of R-D channel still obtains diversity gain for the destination

and block combining at destination, which are implemented in the partial transmission (PT) cooperative strategy. We analyzed the performance of $\mathrm{PT}$, mainly the approximate outage probability of the entire system.

\subsection{Block processing implementation}

In the block processing, we designed a bisearch method to optimize the block size, which aims to obtain the optimal system performance under the power limitation of each relay. The relay calculates the error probability of each block based on soft information. After block selection based on the channel state, the relay forwards a fraction of received message, where the partial transmission at relay is achieved.

\subsection{Block combining implementation}

The destination received several copies of signal in the different time slots. After decoding the signal, the imaginary parts of the signal are used to locate the information block and the real parts are synchronized. The information sequence blocks are combined as one information sequence according to their location, then decoded in the final step.

\subsection{Outage probability calculation}

The outage probability analysis shows the performance of cooperative strategies. Assuming every bit consumes the equal power, the calculation depends on SNR of the transmitted block numbers. We used the Laplace transform to approximate the multiple integral of outage probability, which is demonstrated in Appendix.

\subsection{Simulation setting}

The simulation is set up to compare outage probabilities of partial transmission (PT) and three conventional cooperative strategies, direction transmission (DT), selective decode-and-forward (SDF), and amplify-and-forward $(\mathrm{AF})$, in the situations with one-relaying system and two-relaying system. With the normalized system power, the simulation shows the comparison of system performances with unreliable relays.

\section{Conclusions}

In this paper, we analyzed the partial transmission cooperative strategy that allows the relays to divide the information into small blocks and only forward the correct blocks. We designed the block processing at relay and block combining at destination to solve the problem of unreliable relays. Relaying processing and block processing compose the system of $\mathrm{PT}$, which is 


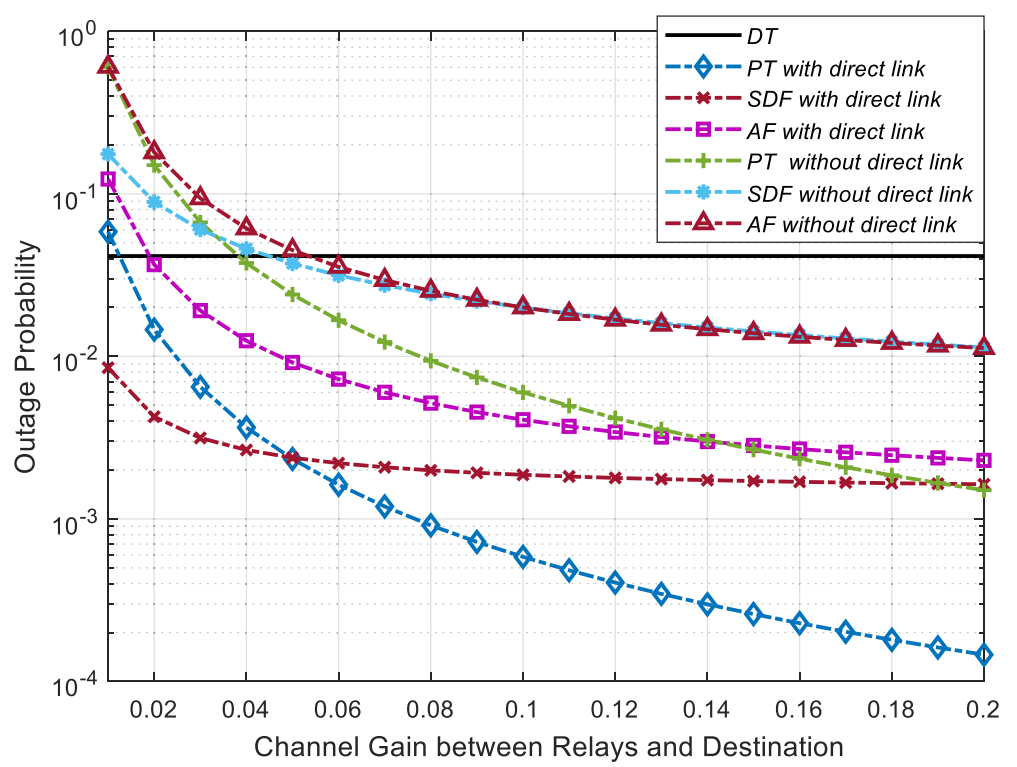

Fig. 8 Outage probability versus channel gain between relay and destination with two relays. The figure shows that the simulations on outage probability versus channel gain between relay and destination (R-D) of partial transmission (PT) and three conventional cooperative strategies, direction transmission (DT), selective decode-and-forward (SDF), and amplify-and-decode (AF), on the situations with two-relaying system. To simulate the unreliable relays, the simulation parameters are set as follows: the number of information bits is 100,000; the transmit SNR of source is $20 \mathrm{~dB}$; other channel states from relays to destination (R-D) and source to destination (S-D) are set the same, as 0.1. In Fig. 8, in order to facilitate the analysis, we assume that the two R-D channels follow the same distribution. As the channel gain between relays and destination increases, the gain value that PT gets better than SDF is nearly half of it in one-relaying system, where the certain amount of location information of PT degrades some performance. Generally speaking, when the R-D channel is better than the severely attenuated S-D channel, i.e., the relays are considered to be unreliable, the performance of PT is better than that of the conventional strategies

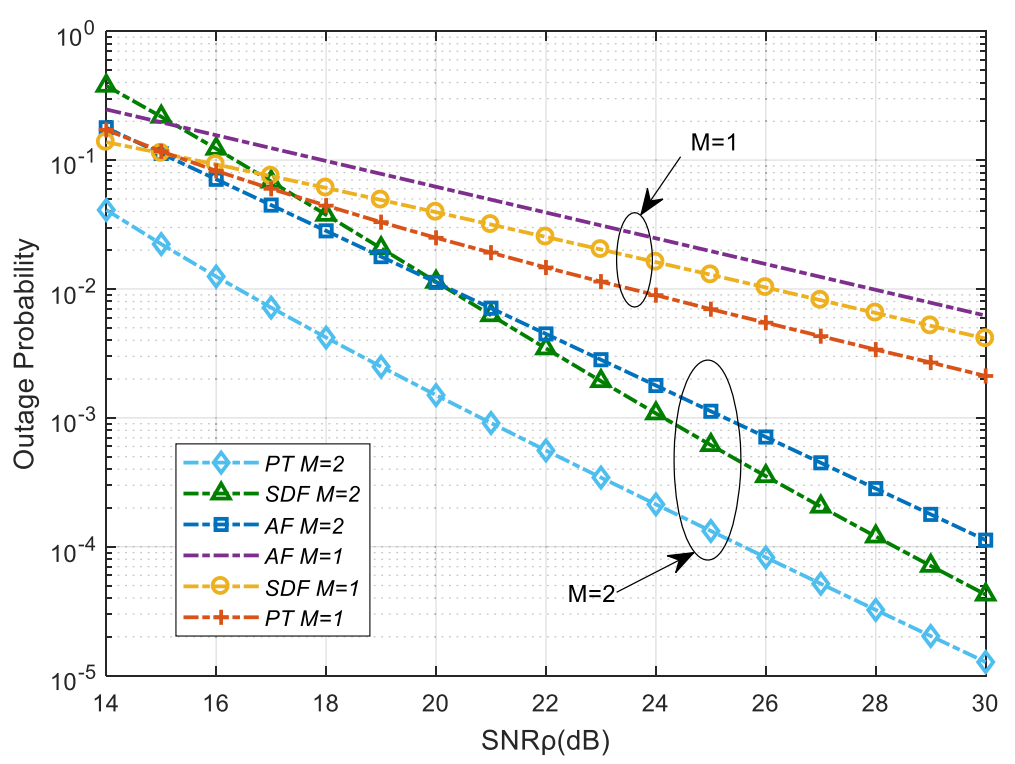

Fig. 9 Outage probability versus channel gain between relay and destination without direct link. The figure shows that the simulations on outage probability versus SNR of partial transmission (PT) and three conventional cooperative strategies, direction transmission (DT), selective decode-andforward (SDF), and amplify-and-decode (AF) without the existence of direct link, on the situations with one-relaying system and two-relaying system. To simulate the unreliable relays, the simulation parameters are set as: the number of information bits is 100,000; the transmission rate of information is $0.5 \mathrm{~b} / \mathrm{s} / \mathrm{Hz}$; the channel states from relays to destination is set as 0.2 , and those from source to relays and source to destination are set the same, as 0.1 . At low SNR, Fig. 9 shows none of the mentioned strategies are reliable. As SNR increases, the improvements of PT are more obvious without direct link. Certainly, the existence of the direct link helps to gain more spatial diversity gain, which can be seen when compared to Fig. 5 
in fact a distributed system allowing the relays to make decisions for destination. We derived the approximate expression of the end-to-end outage probability as a function of SNR, the information transmission rate, S-R channel gain, and the number of relays. Simulations are made in one-relaying system and two-relaying system, which are also carried out in continuous change of the S-R channel. We found that the performance of PT gets overwhelming in unreliable-relaying system. Sequentially, we noted that PT can better benefit from the R-D channel, and the higher the information transmission rate is, the better performance PT can get. Extensive numerical and simulation studies illustrate our theoretical developments and show that the proposed strategy provides a significant improvement over the conventional cooperative strategies on unreliable relays.

\section{Appendix}

In this Appendix, we prove (19) and (20). When $x$ is a Nakagami RV with Nakagami parameter $m_{i}$ and $\mathrm{E}\left(x^{2}\right)$ $=\rho \bar{\gamma}$, then the PDF of $x$ is:

$$
f_{\mathrm{SNR}}(x)=\frac{2 m_{i}}{(\rho \bar{\gamma})^{\mathrm{m}_{\mathrm{i}}} \cdot \Gamma\left(m_{i}\right)} \cdot x^{2 m_{i}-1} \cdot \exp \left(-\frac{m_{i} x^{2}}{\rho \bar{\gamma}}\right) \quad x \geq 0
$$

Assuming:

$$
Z=\mathrm{SNR}_{\mathrm{sd}}+\sum_{i=1}^{M} \mathrm{SNR}_{\mathrm{r}_{i} \mathrm{~d}}
$$

(19a) can be expressed as:

$$
\begin{aligned}
\operatorname{Prob}\left(I_{\mathrm{PT}}<R_{\mathrm{t}}\right) & =\operatorname{Prob}\left(\frac{1}{1+M} \log _{2}\left(1+\mathrm{SNR}_{\mathrm{sd}}+\sum_{i=1}^{M} \mathrm{SNR}_{\mathrm{r}_{\mathrm{i}} \mathrm{d}}\right)<R_{\mathrm{t}}\right) \\
& =\operatorname{Prob}\left(\mathrm{SNR}_{\mathrm{sd}}+\sum_{i=1}^{M} \mathrm{SNR}_{\mathrm{r}_{i} \mathrm{~d}}<2^{(M+1) R_{\mathrm{t}}}-1\right) \\
& =\operatorname{Prob}(Z<\alpha)
\end{aligned}
$$

where $\alpha=2^{(M+1) R_{\mathrm{t}}}-1$. The Laplace transform of the PDF of $\mathrm{f}_{\mathrm{SNR}}(x)$ is:

$$
\mathcal{L}_{x^{2}}(s)=\left(\frac{m_{i}}{m_{i}+\rho \bar{\gamma} \cdot s}\right)^{m_{i}}
$$

The Laplace transform of the PDF of $Z$ in (32) is

$$
\mathcal{L}_{Z}(s)=\prod_{i=1}^{M}\left(\frac{m_{i}}{m_{i}+\rho \bar{\gamma} \cdot s}\right)^{m_{i}}
$$

For large $\rho$, we can approximate (34) as

$$
\mathcal{L}_{x^{2}}(s) \approx\left(\frac{m_{i}}{\rho \bar{\gamma}}\right)^{m_{i}} \cdot \frac{1}{s^{m_{i}}}
$$

Then an approximation of (35) is

$$
\mathcal{L}_{Z}(s) \approx \frac{1}{s\left[\sum_{i=1}^{M} m_{i}\right]} \times \prod_{i=1}^{M}\left(\frac{m_{i}}{\rho \bar{\gamma}}\right)^{m_{i}}
$$

The inverse Laplace transform of $\mathcal{L}_{Z}(\mathrm{~s})$ gives us an approximation to the PDF of $Z$ as:

$$
f_{Z}(z) \approx \frac{z^{\left[\sum_{i=1}^{M} m_{i}-1\right]}}{\Gamma\left(\sum_{i=1}^{M} m_{i}\right)} \times \prod_{i=1}^{M}\left(\frac{m_{i}}{\rho \bar{\gamma}}\right)^{m_{i}}
$$

Therefore, the probability that $Z$ is less than $\alpha$ is

$$
\operatorname{Prob}(Z<\alpha) \approx \frac{\alpha\left[\sum_{i=1}^{M} m_{i}-1\right]}{\Gamma\left(\sum_{i=1}^{M} m_{i}\right)} \prod_{i=1}^{M}\left(\frac{m_{i}}{\rho \bar{\gamma}}\right)^{m_{i}}
$$

Using (33) and (39), after RV equivalence replacement, we obtain:

$$
\begin{aligned}
\operatorname{Prob}\left(I_{\mathrm{PT}}<R_{\mathrm{t}}\right) \approx & \frac{\left(2^{(1+M) R_{\mathrm{t}}}-1\right)^{\left[\sum_{i=1}^{M} m_{i}+m_{\mathrm{sd}}\right]}}{\Gamma\left(1+\sum_{i=1}^{M} m_{i}+m_{\mathrm{sd}}\right)} \cdot\left(\frac{m_{\mathrm{sd}}}{\rho \bar{\gamma}}\right)^{m_{\mathrm{sd}}} \prod_{i=1}^{M} \\
& \times\left(\frac{m_{i} N}{\rho\left\lfloor N \cdot\left(1-\mathrm{BER} \mathrm{sr}_{i}\right)\right\rfloor \bar{\gamma}_{\mathrm{r}_{\mathrm{i}} \mathrm{d}}}\right)^{m_{i}}
\end{aligned}
$$

where $m_{\mathrm{sd}}$ is the Nakagami parameter of the direct link. If without the direct link, (40) turns into:

$$
\begin{aligned}
\operatorname{Prob}\left(I_{\mathrm{PT}}<R_{\mathrm{t}}\right) \approx & \frac{\left(2^{M \cdot R_{\mathrm{t}}}-1\right)^{\sum_{i=1}^{M} m_{i}}}{\Gamma\left(1+\sum_{i=1}^{M} m_{i}\right)} \prod_{i=1}^{M} \\
& \times\left(\frac{m_{i} N}{\rho\left\lfloor N \cdot\left(1-\mathrm{BER}_{\mathrm{sr}_{i}}\right)\right\rfloor \bar{\gamma}_{\mathrm{r}_{i} \mathrm{~d}}}\right)^{m_{i}}
\end{aligned}
$$

\section{Abbreviations}

AF: Amplify-and-forward; CSI: Channel state information; DT: Direct transmission; PT: Partial transmission; RV: Random variable; SDF: Selective decode-and-forward; SISO: Soft input soft output; SNR: Signal and noise ratio

Funding

This work is supported in part by the 863 High Technology Plan of China (2013AA013402) and the National Natural Science Foundation of China $(61172080,61771357)$

\section{Authors' contributions}

$Y L$ and TS are the main writers of this work. They proposed the main idea and described the whole relaying process. $Y L$ derived the formulas. $Y L, Y G$, and ZD performed the simulations and edited the manuscript. All authors read and approved the final manuscript. 


\section{Publisher's Note}

Springer Nature remains neutral with regard to jurisdictional claims in published maps and institutional affiliations.

Received: 30 May 2018 Accepted: 30 July 2018

Published online: 10 August 2018

\section{References}

1. T Cover, AE Gamal, Capacity theorems for the relay channel. IEEE Trans. Info. Theory 25(5), 572-584 (1979)

2. JN Laneman, DNC Tse, GW Wornell, Cooperative diversity in wireless networks: efficient protocols and outage behavior. IEEE Trans. Inf. Theory 50(12), 3062-3080 (2004)

3. $L$ Yang, J Chen, $H$ Jiang, SA Vorobyov, $H$ Zhang, Optimal relay selection for secure cooperative communications with an adaptive eavesdropper. IEEE Trans. Wirel. Commun. 16(1), 26-42 (2017)

4. X Zhou, M Cheng, X He, T Matsumoto, Exact and approximated outage probability analyses for decode-and-forward relaying system allowing intralink errors. IEEE Trans. Wirel. Commun. 13(12), 7062-7071 (2014)

5. Z Mo, W Su, S Batalama, et al., Cooperative communication protocol designs based on optimum power and time allocation. IEEE Trans. Wirel. Commun. 13(8), 4283-4296 (2014)

6. Y Li, MS Rahman, SX Ng, B Vucetic, Distributed soft coding with a soft input soft output (SISO) relay encoder in parallel relay channels. IEEE Trans. Commun. 61(9), 3660-3672 (2013)

7. WK Sang, Cooperative communications with unreliable relays. IEEE Trans, Wirel. Commun. 13(11), 5932-5939 (2014)

8. Z Zhou, M Peng, Z Zhao, W Wang, RS Blum, Wireless-powered cooperative communications:power-splitting relaying with energy accumulation. IEEE J. Sel. Areas Commun. 34(4), 969-982 (2016)

9. R Pabst, BH Walke, DC Schultz, et al., Relay-based deployment concepts for wireless and mobile broadband radio. IEEE Commun. Mag. 42(9), 80-89 (2004)

10. V Genc, S Murphy, Y Yu, et al., IEEE 802.16J relay-based wireless access networks: an overview. IEEE Wirel. Commun. 15(5), 56-63 (2008)

11. D. Schultz, L. Coletti, K. Navaie, et al. Relaying concepts and supporting actions in the context of CGs, IST-4-027756 WINNER II, D3.5.1, v1.0, 2006

12. K. Doppler, S. Redana, K. Schultz, et al. Assessment of relay based deployment concepts and detailed description of multi-hop capable RAN protocols as input for the concept group work, IST-4-027756 WINNER II, D3. 5.2, v1.0, 2006

13. C Abourjeily, Performance analysis of selective relaying in cooperative freespace optical systems. J. Lightw. Technol 31(18), 2965-2973 (2013)

14. ND Chatzidiamantis, DS Michalopoulos, EE Kriezis, et al., Relay selection protocols for relay-assisted free-space optical systems. IEEE J. Opt. Commun. Netw. 5(1), 92-103 (2013)

15. Sophia Antipolis, France, Further advancements for E-UTRA; Physical layer aspects, 3GPP TR 36.814 V9.0.0, Release 9, 2010

16. Hadjtaieb A, Chelli A, Alouini M S. On the performance of hybrid ARQ with incremental redundancy over amplify-and-forward dual-hop relay networks, in Proc IEEE VTC2014-Fall, Vancouver, BC, 2014, pp. 1-5.

17. GGDO Brante, RD Souza, ME Pellenz, Cooperative partial retransmission scheme in incremental decode-and-forward relaying. EURASIP J. Wireless Commun. Netw. 1, 57 (2011)

18. J He, V Tervo, S Qian, M Juntti, T Matsumoto, Performance analysis of OSTBC transmission in lossy forward MIMO relay networks. IEEE Trans. Commun. Lett. 21(8), 1791-1794 (2017)

19. E.E. Tsiropoulou, G. Mitsis, S. Papavassiliou, et al. Interest-aware energy collection \& resource management in machine to machine communications, in Elsevier, Ad Hoc Netw., vol. 68, pp. 48-57, 2018,

20. EE Tsiropoulou, ST Paruchuri, JS Baras, Interest, energy and physical-aware coalition formation and resource allocation in smart loT applications, 2017 51st Annual Conference on Information Sciences and Systems (CISS) (2017), pp. 1-6

21. AR Raghavan, CW Baum, A reliability output Viterbi algorithm with applications to hybrid ARQ. IEEE Trans. Info. Theory 44, 1214-1216 (1998)

22. JC Fricke, PA Hoeher, Reliability-based retransmission criteria for hybrid ARQ. IEEE Trans. Commun. 57(8), 2181-2184 (2009)

\section{Submit your manuscript to a SpringerOpen ${ }^{\circ}$ journal and benefit from:}

- Convenient online submission

- Rigorous peer review

- Open access: articles freely available online

- High visibility within the field

- Retaining the copyright to your article

Submit your next manuscript at $\boldsymbol{\nabla}$ springeropen.com 